Anal. Theory Appl.

Vol. 28, No. 1 (2012), 27-37

\title{
THE HAUSDORFF MEASURE OF SIERPINSKI CARPETS BASING ON REGULAR PENTAGON*
}

\author{
Chaoyi Zeng, Dehui Yuan \\ (Hanshan Normal University, China) \\ and \\ Shaoyuan $\mathrm{Xu}^{* *}$ \\ (Gannan Normal University, China)
}

Received Jan. 19, 2011

\begin{abstract}
In this paper, we address the problem of exact computation of the Hausdorff measure of a class of Sierpinski carpets E- the self-similar sets generating in a unit regular pentagon on the plane. Under some conditions, we show the natural covering is the best one, and the Hausdorff measures of those sets are euqal to $|E|^{s}$, where $s=\operatorname{dim}_{H} E$.
\end{abstract}

Key words: Sierpinski carpet, Hausdorff measure, upper convex density

AMS (2010) subject classification: $28 \mathrm{~A} 78,28 \mathrm{~A} 80$

\section{Introduction}

The Hausdorff measure and dimension are the most important concepts in fractal geometry, and their computation is very difficult. Recently, in order to study deeply the Hausdorff measure, the reference [1] gave the notions "best covering" and "natural covering", and posed eight open problems and six conjectures on Hausdorff measure. Using the notion upper convex density of a class of self-similar sets, the reference [2] studied a class of self-similar sets-generating in a unit square on the plane, proved that the natural covering is the best one and the Hausdorff measures of those sets are euqal to $\sqrt{2}^{s}$. In this paper, we address the problem of the exact computation

* Partially supported by National Natural Science Foundation of China (No. 10961003).

Corresponding author. 
of the Hausdorff measure of a class self similar sets-generating in a unit regular pentagon on the plane.

For convenience, we first present some notions that will be used in the rest part of the paper.

Definition 1. Suppose $E \subset \mathbf{R}^{2}, s \in \mathbf{R}, s \geq 0$ and $\delta>0$, the Hausdorff measure of the set $E$ is defined as

$$
H^{s}(E)=\lim _{\delta \rightarrow 0} \inf \left\{\sum_{i=1}^{\infty}\left|U_{i}\right|^{s}:\left|U_{i}\right| \leqslant \delta, E \subset \bigcup U_{i}\right\}
$$

where $\left\{U_{i}\right\}_{i=1}^{\infty}$ is arbitrary covering of the set $E$; and the Hausdorff dimension of $E$ (denoted by $\operatorname{dim}_{H} E$ ) is defined as

$$
\operatorname{dim}_{H} E=\sup \left\{s: H^{s}(E)=\infty\right\}=\inf \left\{s: H^{s}(E)=0\right\}
$$

Definition 2. Let $\delta>0, s \geq 0, E \subset R^{2}, x \in E$. Moreover, for a convex set $U_{x}$ containing $x$, the upper convex density of $E$ at $x$ is defined as

$$
\bar{D}_{C}^{s}(E, x)=\lim _{\delta \rightarrow 0} \sup _{0<\left|U_{x}\right|<\delta}\left\{\frac{H^{s}\left(E \cap U_{x}\right)}{\left|U_{x}\right|^{s}}\right\} .
$$

The properties of the upper convex density are discussed in the reference [5].

Definition 3. (See Fig. 1) Let $E_{0}$ be an unit regular pentagon $A_{1} A_{2} A_{3} A_{4} A_{5}$ on the plane $R^{2}$, $E$ be the attractor generated by the iterated function system (IFS) $\left\{f_{i} \mid i=1,2,3,4,5\right\}$, where

$$
\begin{aligned}
& f_{i}(x)=\lambda_{i} x+b_{i}, 0<\lambda_{i}<1, i=1,2,3,4,5, x=\left(x_{1}, x_{2}\right) \in E_{0} \\
& b_{1}=\left(\left(1-\lambda_{1}\right) \sin 18^{\circ}, 0\right) \\
& b_{2}=\left(\left(1-\lambda_{2}\right)\left(\sin 18^{\circ}+1\right), 0\right) \\
& b_{3}=\left(\left(1-\lambda_{3}\right)\left(2 \sin 18^{\circ}+1\right),\left(1-\lambda_{3}\right) \cos 18^{\circ}\right) \\
& b_{4}=\left(\left(1-\lambda_{4}\right) \cos 18^{\circ},\left(1-\lambda_{4}\right)\left(\sin 18^{\circ}+\cos 18^{\circ}\right)\right) \\
& b_{5}=\left(0,\left(1-\lambda_{5}\right) \cos 18^{\circ}\right)
\end{aligned}
$$

Then the self-similar set $E$ is called a Sierpinski carpet generating in a unit regular pentagon, where $s=\operatorname{dim}_{H} E$ satisfies $\sum_{i=1}^{5} \lambda_{i}^{s}=1$.

\section{Two Lemmas}

In this section, we present two lemmas which will be used in the proof of the main result of this paper. 


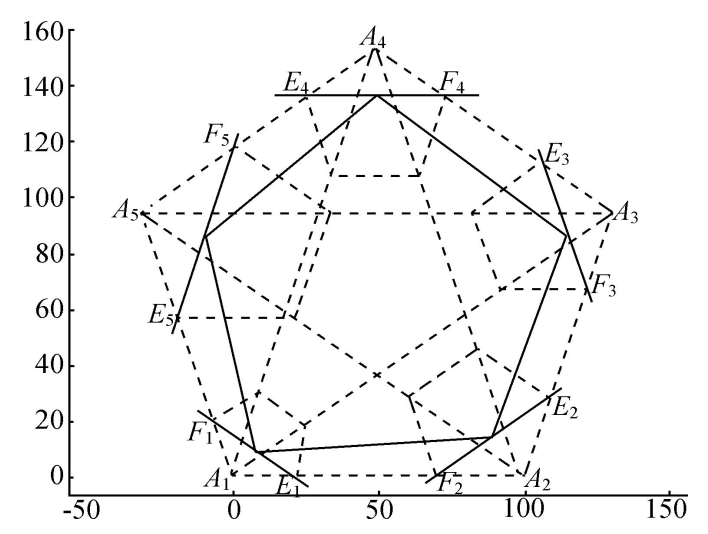

Fig. 1

From the definition 3, it is easy to see that

$$
\bigcup_{i=1}^{5} f_{i}\left(E_{0}\right) \subset E_{0} \quad \text { and } \quad \bigcup_{i=1}^{5} f_{i}(E)=E .
$$

Let $\mu$ be the unique probability measure satisfying the self-similar relation and

$$
\mu=\sum_{i=1}^{5} \lambda_{i}^{s} \mu f_{i}^{-1}
$$

then $E$ is the support of $\mu$ and $\mu$ is a mass distribution on $E$.

For $i=1,2,3,4,5$, let $E_{i} F_{i}$ be parallel to the opposite side of vertex $A_{i}$ in an unit regular pentagon and intersect $f_{i}(E)$, let $d_{i}=\operatorname{dist}\left(A_{i}, E_{i} F_{i}\right)$ be the distance between point $A_{i}$ and line $E_{i} F_{i}$. Denote

$$
t_{i}=\frac{d_{i}}{\cos 18^{\circ}}
$$

if

$$
0<\lambda_{i}<\frac{7-2 \sqrt{5}}{9}
$$

and $0<d_{i}<(\sqrt{5}+1) \lambda_{i}$, then the line $E_{i} F_{i}$ doesn't intersect $f_{j}(E)$ for $i, j \in\{1,2,3,4,5\}$ and $i \neq j$. Assume that $\mu\left(t_{i}\right)$ is the measure of the triangle $\Delta A_{i} E_{i} F_{i}$. Moreover, we use the notations:

$$
\begin{gathered}
d\left(\mu, t_{i}\right)=\frac{\mu\left(t_{i}\right)}{t_{i}^{s}}, d_{\min }^{(i)}=\inf _{0<t_{i} \leq 2 \sin 54^{\circ}}\left\{d\left(\mu, t_{i}\right)\right\}, i=1,2,3,4,5 \\
M_{1}=\left\{(i, j) \mid A_{i} A_{j} \text { is the diagonal of } E_{0}, i, j=1,2,3,4,5\right\}
\end{gathered}
$$


and

$$
M_{2}=\left\{(i, j) \mid A_{i} A_{j} \text { is the side of } E_{0}, i, j=1,2,3,4,5\right\} .
$$

That is

$$
M_{1}=\{(1,3),(3,1),(1,4),(4,1),(2,4),(4,2),(2,5),(5,2),(3,5),(5,3)\}
$$

and

$$
M_{2}=\{(1,2),(2,1),(2,3),(3,2),(3,4),(4,3),(4,5),(5,4),(1,5),(5,1)\} .
$$

Lemma 4. Let

$$
0<\lambda_{i}<\frac{7-2 \sqrt{5}}{9}, \quad 0<s<1, \quad i=1,2,3,4,5 .
$$

Assume that $K$ is a nonnegative integer. Then $d\left(\mu, t_{i}\right)$ attains its infimum $d_{\min }^{(i)}$ only at the following values of $t_{i}$ :

(1) $t_{i}=\lambda_{i}^{K} \frac{1-\lambda_{p}}{2 \sin 18^{\circ}},(i, p) \in M_{1}$, or

(2) $t_{i}=\lambda_{i}^{K} \cdot \min _{i \neq q, p \neq q}\left\{2 \sin 18^{\circ}\left(1-\lambda_{q}\right)\right\},(i, q) \in M_{2}$.

Proof. Since $E$ is self-similar, then we need only prove that the result is true when $2 \lambda_{1} \sin 54^{\circ}<$ $t_{1} \leq 2 \sin 54^{\circ}$ for $i=1$. Denote

$$
\begin{aligned}
& r_{1}=\lambda_{1} \sin 54^{\circ}, r_{2}=\min \left\{2\left(1-\lambda_{2}\right) \sin 18^{\circ}, 2\left(1-\lambda_{5}\right) \sin 18^{\circ}\right\}, \\
& r_{3}=2 \sin 54^{\circ}-\min \left\{2\left(1-\lambda_{2}\right) \sin 18^{\circ}, 2\left(1-\lambda_{5}\right) \sin 18^{\circ}\right\}, \\
& r_{3}=2 \sin 54^{\circ}-\max \left\{2 \lambda_{3} \sin 54^{\circ}, 2 \lambda_{4} \sin 54^{\circ}\right\} .
\end{aligned}
$$

Case 1. $d\left(\mu, t_{1}\right)$ attains its infimum $d_{\min }^{(1)}$ at the interval $\left(r_{2}, r_{3}\right]$. In this case, the line $E_{1} F_{1}$ intersects $f_{2}(E)$ or $f_{5}(E)$. Therefore

$$
\begin{aligned}
& d\left(\mu, t_{1}\right)=\frac{\mu\left(t_{1}\right)}{t_{1}^{s}} \\
& =\frac{\lambda_{1}^{s}+\mu\left(t_{1}-2\left(1-\lambda_{2}\right) \sin 18^{\circ}\right)+\mu\left(t_{1}-2\left(1-\lambda_{5}\right) \sin 18^{\circ}\right)}{\left(r_{2}+t_{1}-r_{2}\right)^{s}} \\
& \geq \frac{\lambda_{1}^{s}+\max \left\{\mu\left(t_{1}-2\left(1-\lambda_{2}\right) \sin 18^{\circ}\right), \mu\left(t_{1}-2\left(1-\lambda_{5}\right) \sin 18^{\circ}\right)\right\}}{\left(r_{2}+t_{1}-r_{2}\right)^{s}} \\
& \geq \frac{\lambda_{1}^{s}+\max \left\{\mu\left(t_{1}-\left(1-\lambda_{2}\right) 2 \sin 18^{\circ}\right), \mu\left(t_{1}-\left(1-\lambda_{5}\right) 2 \sin 18^{\circ}\right)\right\}}{r_{2}^{s}+\left(t_{1}-r_{2}\right)^{s}} \\
& \geq \min \left\{\frac{\lambda_{1}^{s}}{r_{2}^{s}}, \frac{\tau}{\left(t_{1}-r_{2}\right)^{s}}\right\},
\end{aligned}
$$

where

$$
\tau=\max \left\{\mu\left(t_{1}-\left(1-\lambda_{2}\right) 2 \sin 18^{\circ}\right), \mu\left(t_{1}-\left(1-\lambda_{5}\right) 2 \sin 18^{\circ}\right)\right\}
$$


This contradicts the assumption that $d\left(\mu, t_{1}\right)$ attains its infimum $d_{\min }^{(1)}$ at the interval $\left(r_{2}, r_{3}\right]$.

Case 2. $d\left(\mu, t_{1}\right)$ attains its infimum $d_{\min }^{(1)}$ at $\left(r_{4}, 2 \sin 54^{\circ}\right]$. In this case, the line $E_{1} F_{1}$ intersects $f_{3}(E)$ or $f_{4}(E)$, then

$$
\begin{aligned}
& d\left(\mu, t_{1}\right)=\frac{\mu\left(t_{1}\right)}{t_{1}^{s}} \\
& =\frac{\lambda_{1}^{s}+\lambda_{2}^{s}+\lambda_{3}^{s}+\max \left\{\mu\left(t_{1}-2 \lambda_{3} \sin 54^{\circ}\right), \mu\left(t_{1}-2 \lambda_{4} \sin 54^{\circ}\right)\right.}{\left(r_{4}+t_{1}-r_{4}\right)^{s}} \\
& \geq \frac{\lambda_{1}^{s}+\lambda_{2}^{s}+\lambda_{3}^{s}+\max \left\{\mu\left(t_{1}-2 \lambda_{3} \sin 18^{\circ}\right), \mu\left(t_{1}-2 \lambda_{4} \sin 18^{\circ}\right)\right.}{\left(r_{4}+t_{1}-r_{4}\right)^{s}} \\
& \geq \frac{\lambda_{1}^{s}+\lambda_{2}^{s}+\lambda_{3}^{s}+\max \left\{\mu\left(t_{1}-2 \lambda_{3} \sin 18^{\circ}\right), \mu\left(t_{1}-2 \lambda_{4} \sin 18^{\circ}\right)\right.}{r_{4}^{s}+\left(t_{1}-r_{4}\right)^{s}} \\
& \geq \min \left\{\frac{\lambda_{1}^{s}}{r_{4}^{s}}, \frac{\lambda_{2}^{s}}{r_{4}^{s}}, \frac{\lambda_{3}^{s}}{r_{4}^{s}}, \frac{\tau}{\left(t_{1}-r_{4}\right)^{s}}\right\},
\end{aligned}
$$

where $\tau=\max \left\{\mu\left(t_{1}-2 \lambda_{3} \sin 54^{\circ}\right), \mu\left(t_{1}-2 \lambda_{4} \sin 18^{\circ}\right)\right\}$.

Case 3. $d\left(\mu, t_{1}\right)$ attains its infimum $d_{\min }^{(1)}$ at $\left(2 \lambda_{1} \sin 54^{\circ}, r_{2}\right]$. In this case, we have

$$
d\left(\mu, t_{1}\right)=\frac{\lambda_{1}^{s}}{t_{1}^{s}} \geq \frac{\lambda_{1}^{s}}{r_{2}^{s}}
$$

This means that $t_{1}=2\left(1-\lambda_{2}\right) \sin 18^{\circ}$ or $t_{1}=2\left(1-\lambda_{5}\right) \sin 18^{\circ}$, and $K=0$.

Case 4. $d\left(\mu, t_{1}\right)$ attains its infimum $d_{\min }^{(1)}$ at the interval $\left(r_{3}, r_{4}\right]$. We have

$$
d\left(\mu, t_{1}\right)=\frac{\lambda_{1}^{s}+\lambda_{2}^{s}+\lambda_{3}^{s}}{t_{1}^{s}} \geq \frac{\lambda_{1}^{s}+\lambda_{2}^{s}+\lambda_{3}^{s}}{r_{4}^{s}}
$$

Therefore,

$$
d\left(\mu, t_{1}\right)=\frac{\lambda_{1}^{s}+\lambda_{2}^{s}+\lambda_{3}^{s}}{r_{4}^{s}}
$$

This means that $t_{1}=\frac{1-\lambda_{3}}{2 \sin 18^{\circ}}$ or $t_{1}=\frac{1-\lambda_{4}}{2 \sin 18^{\circ}}$, and $K=0$.

Similarly, if $K>0$ and $2 \lambda_{1}^{k+1} \sin 54^{\circ}<t_{1}<\lambda_{1}^{k} \sin 54^{\circ}$, we can prove that $d\left(\mu, t_{1}\right)$ attains its infimum $d_{\min }^{(1)}$ only at $t_{1}=\lambda_{1}^{k} \frac{1-\lambda_{3}}{2 \sin 18^{\circ}}, \lambda_{1}^{k} \frac{1-\lambda_{4}}{2 \sin 18^{\circ}}, 2 \lambda_{1}^{k}\left(1-\lambda_{2}\right) \sin 18^{\circ}$ and $2 \lambda_{1}^{k}\left(1-\lambda_{5}\right) \sin 18^{\circ}$.

Lemma $5^{[3]}$. Let $0<\alpha<1, p \leq p_{0}, a \geq a_{0}, y \geq \lambda x^{\alpha}$. If

$$
0<x \leq\left(\frac{a_{0} \lambda}{p_{0}}\right)^{\frac{1}{1-\alpha}},
$$

then $\frac{p-y}{(a-x)^{\alpha}}<\frac{p}{a^{\alpha}}$. 


\section{The Main Result}

Theorem 6. Let $E$ be a self-similar set defined by Definition 3, $0<\lambda_{i}<\frac{7-2 \sqrt{5}}{9}(i=$ $1,2,3,4,5), s=\operatorname{dim}_{H}(E)$ and $0<s<1$. Moreover, assume the following two conditions

$$
\begin{aligned}
& \text { (1) } \frac{\lambda_{i}^{s}+\lambda_{j}^{s}}{\left(1-\lambda_{i}-\lambda_{j}\right)^{s}} \leq\left(\frac{1}{2 \sin 54^{\circ}}\right)^{s}, \text { for }(i, j) \in M_{2} ; \\
& \text { (2) } 2\left(\lambda_{i}+\lambda_{j}\right) \sin 54^{\circ} \leq \min \left\{2 d_{\min }^{(i)} \sin 54^{\circ}, 2 d_{\min }^{(j)} \sin 54^{\circ}\right\}^{\frac{1}{1-s}}, \\
& \quad \text { for }(i, j) \in M_{1}
\end{aligned}
$$

are satisfied. Then for any $x \in E$, if the closed convex set $U_{x}$ containing $x$ is the closure $\bar{E}_{0}$ of $E_{0}$, then

$$
\bar{D}_{C}^{s}(E, x)=\sup _{0<\left|U_{x}\right|}\left\{\frac{H^{s}\left(E \cap U_{x}\right)}{\left|U_{x}\right|^{s}}\right\}=\frac{H^{s}\left(E \cap \bar{E}_{0}\right)}{\left(2 \sin 54^{\circ}\right)^{s}}=1 .
$$

Proof. Let $V \subset R^{2}, V \cap E \neq \varnothing$ and $V \subset \bar{E}_{0}$ (if not, replacing $V$ by $V \cap \bar{E}_{0}$ ). Denote

$$
d(V)=\frac{\mu(V)}{|V|^{s}}, d_{\max }=\sup _{0<|v|}\left\{d(V), V \subset \bar{E}_{0}\right\},
$$

where $\mu$ is the mass distribution of $E$ defined as above. We now prove that if $V=\bar{E}_{0}$ then

$$
d_{\max }=\frac{\mu(V)}{|V|^{s}}=\frac{\mu\left(\bar{E}_{0}\right)}{\left|\bar{E}_{0}\right|^{s}} .
$$

Case 1. $V \cap f_{i}(E) \neq \varnothing$ for all $i$ (See Fig. 2). In this case, we can select fine tangent

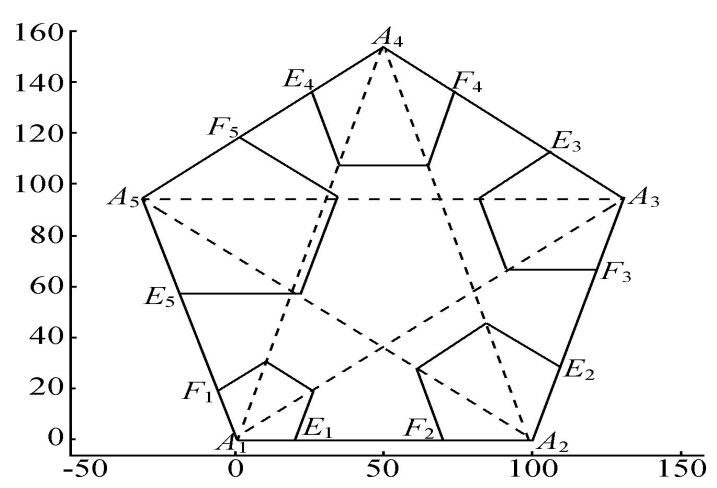

Fig. 2

lines of $V$, denoted by $E_{i} F_{i}$, such that $E_{i} F_{i}$ is parallel to the opposite side of the vertex $A_{i}$ for $i=1,2,3,4,5$. Moreover, denote

$$
t_{i}=\frac{d_{i}}{\cos 18^{\circ}}
$$


where $d_{i}$ is the distance between the vertex $A_{i}$ and $E_{i} F_{i}$, then

$$
\begin{aligned}
& |V| \geq 2 \sin 54^{\circ}-t_{i}-t_{j}, \text { for }(i, j) \in M_{1} \\
& \mu(V) \leq \sum_{i=1}^{5}\left(\lambda_{i}^{s}-\mu\left(t_{i}\right)\right)=1-\sum_{i=1}^{5} \mu\left(t_{i}\right)
\end{aligned}
$$

Therefore

$$
\frac{\mu(V)}{|V|^{s}} \leq \frac{1-\sum_{i=1}^{5} \mu\left(t_{i}\right)}{\left(2 \sin 54^{\circ}-t_{i}-t_{j}\right)^{s}} \leq \frac{1-\left(\mu\left(t_{i}\right)+\mu\left(t_{j}\right)\right)}{\left(2 \sin 54^{\circ}-t_{i}-t_{j}\right)^{s}} .
$$

Replacing $\alpha$ by $s, a$ and $a_{0}$ by $2 \sin 54^{\circ}, p$ and $p_{0}$ by 1 , respectively, in Lemma 5, employing Lemma 4, we have

$$
\begin{aligned}
\frac{\mu\left(t_{i}\right)+\mu\left(t_{j}\right)}{\left(t_{i}+t_{j}\right)^{s}} & \geq \frac{\mu\left(t_{i}\right)+\mu\left(t_{j}\right)}{t_{i}^{s}+t_{j}^{s}} \geq \min \left\{\frac{\mu\left(t_{i}\right)}{t_{i}^{s}}, \frac{\mu\left(t_{j}\right)}{t_{j}^{s}}\right\} \\
& \geq \min \left\{d_{\text {min }}^{(i)}, d_{\min }^{(j)}\right\} . \triangleq \lambda
\end{aligned}
$$

Notice the condition (2), we have

$$
\begin{aligned}
0<\lambda_{i}+\lambda_{j} & \leq \frac{\min \left\{2 d_{\min }^{(i)} \sin 54^{\circ}, 2 d_{\min }^{(j)} \sin 54^{\circ}\right\}^{\frac{1}{1-s}}}{2 \sin 54^{\circ}} \\
& =\left[\left(2 \sin 54^{\circ}\right)^{s}\right]^{\frac{1}{1-s}} \min \left\{d_{\min }^{(i)}, d_{\min }^{(j)}\right\}^{\frac{1}{1-s}} \\
& \leq\left[\left(2 \sin 54^{\circ}\right)\right]^{\frac{1}{1-s}} \min \left\{d_{\min }^{(i)}, d_{\min }^{(j)}\right\}^{\frac{1}{1-s}}=\left(\frac{a_{0} \lambda}{p_{0}}\right)^{\frac{1}{1-s}} .
\end{aligned}
$$

This means that the conditions of Lemma 5 are satisfied. Denote $w=\lambda_{i}+\lambda_{j}$, then

$$
y=\mu\left(t_{i}\right)+\mu\left(t_{j}\right) \geq \lambda\left(t_{i}^{s}+t_{j}^{s}\right) \geq \lambda\left(t_{i}+t_{j}\right)^{s}=\lambda w .
$$

Therefore,

$$
\frac{\mu(V)}{|V|^{s}} \leq \frac{p-y}{(a-w)^{\alpha}} \leq \frac{1}{\left(2 \sin 54^{\circ}\right)^{s}}
$$

That is

$$
d_{\max }=\frac{\mu(V)}{|V|^{s}}=\frac{\mu\left(\bar{E}_{0}\right)}{\left|\bar{E}_{0}\right|^{s}} .
$$

Case 2. There exist only four of five sets $f_{i}(E)$ such that $V \cap f_{i}(E) \neq \varnothing$. For convenience, let $f_{1}(E), f_{2}(E), f_{3}(E)$ and $f_{4}(E)$ be these four sets. Then

$$
\begin{aligned}
& |V| \geq 2 \sin 54^{\circ}-t_{1}-t_{3}, \\
& |V| \geq 2 \sin 54^{\circ}-t_{1}-t_{4}, \\
& |V| \geq 2 \sin 54^{\circ}-t_{2}-t_{4},
\end{aligned}
$$


and

$$
\begin{aligned}
& \mu(V) \leq 1-\mu\left(t_{1}\right)-\mu\left(t_{3}\right), \\
& \mu(V) \leq 1-\mu\left(t_{1}\right)-\mu\left(t_{4}\right), \\
& \mu(V) \leq 1-\mu\left(t_{2}\right)-\mu\left(t_{4}\right) .
\end{aligned}
$$

So,

$$
\begin{gathered}
|V| \geq 2 \sin 54^{\circ}-t_{i}-t_{j} \\
\mu(V) \leq 1-\mu\left(t_{i}\right)-\mu\left(t_{j}\right)
\end{gathered}, \text { for }(i, j) \in M_{3},
$$

where $M_{3} \triangleq M_{1} \backslash\{(2,5),(5,2),(3,5),(5,3)\}$.

Therefore,

$$
\frac{\mu(V)}{|V|^{s}} \leq \frac{1-\left(\mu\left(t_{i}\right)+\mu\left(t_{j}\right)\right)}{\left(2 \sin 54^{\circ}-t_{i}-t_{j}\right)^{s}},(i, j) \in M_{3} .
$$

Employing Lemma 5, we get

$$
\frac{\mu(V)}{|V|^{s}} \leq \frac{1}{\left(2 \sin 54^{\circ}\right)^{s}}
$$

That means that the result is still true.

Case 3. There exist only three of five sets $f_{i}(E)$ such that $V \cap f_{i}(E) \neq \varnothing$. In this case, there exists $\left(i_{0}, j_{0}\right) \in M_{1}$ such that

$$
V \bigcap f_{i_{0}}(E) \neq \varnothing \text { and } V \bigcap f_{j_{0}}(E) \neq \varnothing \text {. }
$$

Similar to the proof of Case 2, we deduce that

$$
\frac{\mu(V)}{|V|^{s}} \leq \frac{1-\left(\mu\left(t_{i_{0}}\right)+\mu\left(t_{j_{0}}\right)\right)}{\left(2 \sin 54^{\circ}-t_{i_{0}}-t_{j_{0}}\right)^{s}} .
$$

This combining with Lemma follows that

$$
\frac{\mu(V)}{|V|^{s}} \leq \frac{1}{\left(2 \sin 54^{\circ}\right)^{s}}
$$

Case 4. There exist only two of five sets $f_{i}(E)$ such that $V \cap f_{i}(E) \neq \varnothing$. Therefore, we can assume that there exists $(i, j)$ such that

$$
V \bigcap f_{i}(E) \neq \varnothing \text { and } V \bigcap f_{j}(E) \neq \varnothing .
$$

If $(i, j) \in M_{1}$, then we get the required result by Case 3. If $(i, j) \in M_{2}$, and assume $(i, j)=(1,2)$, then

$$
\mu(V) \leq \lambda_{1}^{s}+\lambda_{2}^{s}-\mu\left(t_{1}\right)-\mu\left(t_{2}\right) \leq \lambda_{1}^{s}+\lambda_{2}^{s}
$$


and

$$
|V| \geq 1-\frac{d_{1}}{\cos 54^{\circ}}-\frac{d_{2}}{\cos 54^{\circ}} \geq 1-\lambda_{1}-\lambda_{2}
$$

From the condition (1), we have

$$
\frac{\mu(V)}{|V|^{s}} \leq \frac{\lambda_{1}^{s}+\lambda_{2}^{s}}{\left(1-\lambda_{1}-\lambda_{2}\right)^{s}} \leq \frac{1}{\left(2 \sin 54^{\circ}\right)^{s}}
$$

Case 5. There exists only one of five sets $f_{i}(E)$ such that $V \cap f_{i}(E) \neq \varnothing$, for example, $V \cap f_{1}(E) \neq \varnothing$. Notice the function of amplification of $f_{1}^{-1}$, and

$$
\frac{\mu\left(V \cap f_{1}(E)\right)}{\left|V \bigcap f_{1}(E)\right|^{s}}=\frac{\mu\left(f_{1}^{-1}\left(\left(V \cap f_{1}(E)\right)\right)\right.}{\lambda_{1}^{-1}\left|V \cap f_{1}(E)\right|^{s}} .
$$

Denote $V^{\prime}=f_{1}^{-1}\left(V \cap f_{1}(E)\right)$, we can assume

$$
V^{\prime} \bigcap f_{i}(E) \neq \varnothing, V^{\prime} \bigcap f_{j}(E) \neq \varnothing
$$

for some $(i, j)$ and the density is invariant, if not, then take $f_{1}^{-1}\left(V^{\prime}\right)$ as $V^{\prime}$. Similar to the proof of above case, we get the required result.

Therefore,

$$
d_{\max }=\frac{\mu(V)}{|V|^{s}}=\frac{\mu\left(\bar{E}_{0}\right)}{\left|\bar{E}_{0}\right|^{s}}
$$

we finish the proof.

By the definition of probability measure, we know that there exists a constant $C$ such that $\mu=C H^{s}$. So

$$
\bar{D}_{C}^{s}(E, x)=\sup _{0<\left|u_{x}\right|}\left\{\frac{H^{s}\left(E \cap U_{x}\right)}{\left|U_{x}\right|^{s}}\right\}
$$

attains the supremum at the set $\bar{E}_{0}$.

Combining Theorem 2.3 in reference [3] and Proposition 2 in reference [4], we get

$$
\bar{D}_{C}^{s}(E, x)=\sup _{0<\left|u_{x}\right|}\left\{\frac{H^{s}\left(E \cap U_{x}\right)}{\left|U_{x}\right|^{s}}\right\}=\frac{H^{s}\left(E \cap \bar{E}_{0}\right)}{\left(2 \sin 54^{\circ}\right)^{s}}=1 .
$$

Employing Theorem 6, we have the following corollary.

Corollary 7. If the assumptions in Theorem 6 are satisfied, then $\bar{E}_{0}$ is the "best covering" of $E$. That is

$$
H^{s}(E)=\left|\bar{E}_{0}\right|^{s}=\left(2 \sin 54^{\circ}\right)^{s},
$$

where $s=\operatorname{dim}_{H}(E)$ satisfies

$$
\sum_{i=1}^{5} \lambda_{i}^{s}=1
$$




\section{Examples}

Example 8. Let $\lambda_{1}=\lambda_{2}=\lambda_{3}=\lambda_{4}=\lambda_{5}=\frac{1}{25}$, then $s=\frac{1}{2}$. Moreover, we have

(1) $\frac{\lambda_{i}^{s}+\lambda_{j}^{s}}{\left(1-\lambda_{i}-\lambda_{j}\right)^{s}}=\frac{2}{23} \approx 0.0870 \leq\left(\frac{1}{2 \sin 54^{\circ}}\right)^{s}=0.7862$, for $(i, j) \in M_{2}$;

(2) for $(i, j) \in M_{1}, 2 \sin 54^{\circ}\left(\lambda_{i}+\lambda_{j}\right) \approx 0.1294$,

$$
\begin{aligned}
& d_{\min }^{(i)}=\left(\frac{2 \sin 54^{\circ}}{24}\right)^{s} \approx 0.2596 \\
& \min \left\{2 \sin 54^{\circ} \cdot d_{\min }^{(i)}, 2 \sin 54^{\circ} \cdot d_{\min }^{(j)}\right\}^{\frac{1}{1-s}} \\
& =\left(\frac{\left(2 \sin 54^{\circ}\right)^{s+1}}{24^{s}}\right)^{\frac{1}{1-s}}=0.1765 .
\end{aligned}
$$

Hence, the assumptions of Theorem 6 are satisfied. Therefore,

$$
H^{s}(E)=\left(2 \sin 54^{\circ}\right)^{s} \approx 1.272
$$

Example 9. Let $\lambda_{1}=\lambda_{3}=\lambda_{5}=\frac{1}{625}, \lambda_{2}=\lambda_{4}=\frac{1}{25}$. Since $\sum_{i=1}^{5} \lambda_{i}=1$, then $2\left(\frac{1}{25}\right)^{s}+$ $3\left(\frac{1}{625}\right)^{s}=1$. Denote $x=\left(\frac{1}{25}\right)^{s}$, then $3 x^{2}+2 x-1=$ and $s=\frac{1}{2} \log _{5} 3$. Then,

(1) $\left(\frac{1}{2 \sin 54^{\circ}}\right)^{s}=\left(\frac{1}{2 \sin 54^{\circ}}\right)^{\frac{1}{2} \log _{5} 3}=0.7862$, and for $(i, j) \in M_{2}$,

$$
\begin{aligned}
\frac{\lambda_{i}^{s}+\lambda_{j}^{s}}{\left(1-\lambda_{i}-\lambda_{j}\right)^{s}} & =\frac{\left(\frac{1}{25}\right)^{s}+\left(\frac{1}{25}\right)^{s}}{\left(1-\frac{1}{25}-\frac{1}{25}\right)^{s}} \approx 0.6859, \\
\text { or } \quad \frac{\lambda_{i}^{s}+\lambda_{j}^{s}}{\left(1-\lambda_{i}-\lambda_{j}\right)^{s}} & =\frac{\left(\frac{1}{25}\right)^{s}+\left(\frac{1}{625}\right)^{s}}{\left(1-\frac{1}{25}-\frac{1}{625}\right)^{s}} \approx 0.4768, \\
\text { or } \quad \frac{\lambda_{i}^{s}+\lambda_{j}^{s}}{\left(1-\lambda_{i}-\lambda_{j}\right)^{s}} & =\frac{\left(\frac{1}{625}\right)^{s}+\left(\frac{1}{625}\right)^{s}}{\left(1-\frac{1}{625}-\frac{1}{625}\right)^{s}} \approx 0.2225 .
\end{aligned}
$$

(2) for $(i, j) \in M_{1}, 2 \sin 54^{\circ}\left(\lambda_{i}+\lambda_{j}\right) \approx 0.0337$ or 0.0052 ,

$$
\begin{aligned}
& d_{\min }^{(1)}=d_{\min }^{(3)}=d_{\min }^{(5)}=\left(\frac{2 \sin 54^{\circ}}{624}\right)^{\frac{1}{2} \log _{5}{ }^{3}} \approx 0.1309, \\
& d_{\min }^{(2)}=d_{\min }^{(4)}=\left(\frac{2 \sin 54^{\circ}}{24}\right)^{\frac{1}{2} \log _{5}{ }^{3}} \approx 0.3982, \\
& \min \left\{2 d_{\min }^{(i)} \sin 54^{\circ}, 2 d_{\min }^{(j)} \sin 54^{\circ}\right\}^{\frac{1}{1-s}} \\
& =\left(\left(2 \sin 54^{\circ}\right) \cdot 0.1309\right)^{\frac{1}{1-s}}=0.0944 .
\end{aligned}
$$


So the assumptions of Theorem 6 are satisfied. Therefore,

$$
H^{S}(E)=\left(2 \sin 54^{\circ}\right)^{S}=\left(2 \sin 54^{\circ}\right)^{\log _{5} \sqrt{3}} \approx 1.183
$$

\section{References}

[1] Zhou, Z. L. and Feng, L., Twelve Open Problems on the Exact Value of the Hausdorff Measure and on Topological Entropy: a Brief Survey of Recent Results, Nonlinearity, 17(2004), 493-502.

[2] Zhu, Z. W., Zhou, Z. L. and Jia, B. G., The Hausdorff Measure and Upper Convex Density of a Class of Self-similar Sets on the Plane, Acta Mathematica Sinica, Chinese Series, 48:3(2005), 535-540.

[3] Ayer, E. and Strichartz, R. S., Exact Hausdorff Measure and Intervals of Maximum Density for Cantor Sets, Trans. Amer. Math. Soc., $351: 9$ (1999), 3725-3741

[4] Zhou, Z. L., Hausdorff Measure of Self-similar Sets-koch cure, Science of China (Ser A), 28:2(1998), 103-107.

[5] Falconer, K. J., The Geometry of Fractal sets, Cambridge University press, Cambridge (1985).

Department of Mathematics and Information Technology

Hanshan Normal University

Chaozhou, 521041

P. R. China

C. Y. Zeng

E-mail: zcy@hstc.ecu.cn

D. H. Yuan

E-mail: ydhlxl@hstc.edu.cn

S. Y. Xu

School of Mathematics and Computer

Gannan Normal University

Ganzhou, 341000

E-mail: xushaoyuan@126.com 